\title{
Development of Amorphous Silicon Solar Cells with Plasmonic Light Scattering
}

\author{
L. J. Crudgington*, T. Rahman, and S. A. Boden.
}

\begin{abstract}
This paper reports the result of simulation and fabrication of the optical effects of metallic nano-particle arrays within amorphous silicon thin-films. A finite-difference time domain approach is used to design and model nano-particle arrays within opto-electronic models of thin-film amorphous silicon. An increase in optical scattering and localized surface plasmon resonance is observed, resulting in an increase in power absorption within the material active region and a reduction in optical reflection from the film surface. It is shown that this enhancement in optical performance depends on the particle size, shape, position within the structure and proximity to the metallic back reflector. Process development of metal-island films on silicon and glass, followed by the fabrication and measurement of amorphous silicon P-I-N devices featuring plasmonic nano-particles is demonstrated; showing an enhancement in-keeping with results achieved using simulation.
\end{abstract}

Index Terms - PECVD, Amorphous, plasmonic, nanoparticles.

\section{INTRODUCTION}

$\mathrm{I}_{\mathrm{s}}^{\mathrm{s}}$ depth understanding of the effects of optical light scattering technologies is essential when designing thin film silicon photovoltaic devices [1]. Despite their reduced energy conversion efficiencies compared to single-crystal silicon based cells, amorphous silicon (a-Si:H) thin film solar cells are a significantly cheaper technology with greater fabrication flexibility due to the reduction of silicon material usage by a factor of ten or more. The ability to deposit on inexpensive, flexible substrates over large areas such as glass or plastic [2] and the possibility of integration into architectural facades [3] further adds to the appeal of thin film silicon.

In order for this technology to achieve significant market share, their energy conversion efficiency values must be increased using an equally inexpensive technique compatible with their fabrication processes. Optical methods of scattering and trapping incident light within the devices have been extensively researched, which conventionally consist of deposition on to textured substrate materials [4], using dielectric confinement to trap light [5] and more recently, the use of self-organised plasmonic nano-particle layers within the device window layer or back reflector, [6-10] using the deposition and subsequent annealing of thin metal films.

Whilst these techniques often yield favourable results, the accurate tailoring of periodic nano-particle arrays to the absorption characteristics of the amorphous silicon material are expected to yield greater increases in performance compared to the randomised particles.

In the second section of this work, the Lumerical FDTD
Solutions TCAD package is used to model the interaction of incident light with nano-particle arrays incorporated in thinfilm hydrogenated amorphous silicon (a-Si:H) materials. The parameter space is fully explored over a wide range of shapes, dimensions, positions within the structure, constituent materials, and proximity to reflective surfaces, and these variations are used to demonstrate the effects of plasmonic nano-particle arrays on device performance.

In the third section, process development is undertaken to determine the fabrication conditions that result in the optimum particle dimensions corresponding to the simulation results. Conditions including deposited metal thickness, anneal temperature and anneal duration are varied and the results are investigated using scanning electron microscopy (SEM). An a-Si:H solar cell is fabricated, whose conditions have been optimised as published elsewhere [11], and the nano-particle arrays are applied to the back reflector of these devices in order to observe if the performance is enhanced.

\section{Simulation}

\section{A. Set-up}

The simulation model is shown in figure 1 , and is constructed to follow the structure of the optimum amorphous P-I-N silicon devices fabricated in previous published work [11]. It consists of the following optical topography:

- Glass Substrate, $\mathrm{SiO}_{2}$ Palik model [14] - Perfectly Matched Layer (PML), 625nm simulation boundaries.

- Amorphous Silicon, sampled data model [11] - 400nm.

- Indium Tin Oxide, FDTD model [13] - 200nm.

- $\mathrm{Al} / \mathrm{Ag}$ Back Reflector, Palik model [14] - 300nm.

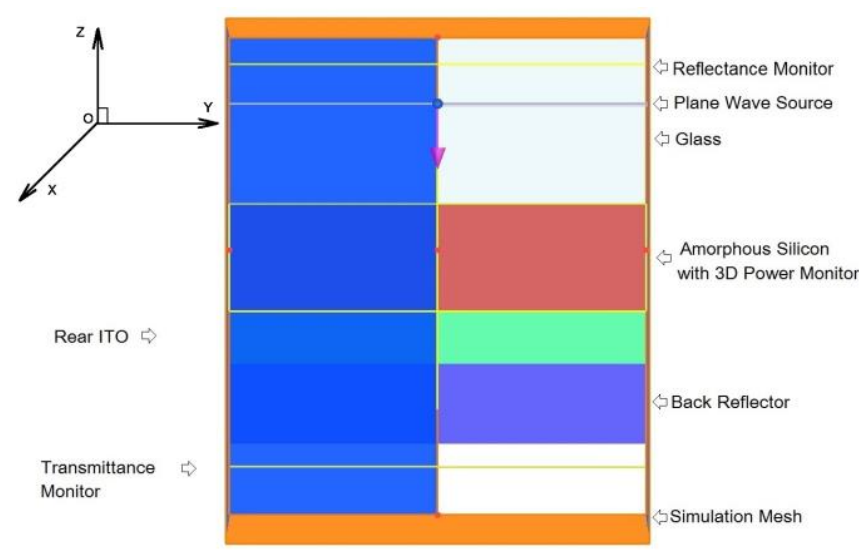

Figure 1 - Schematic of cross-section of the typical device simulation layout 
The simulation boundaries are specified as $400 \mathrm{~nm}$ in the $\mathrm{x}$ and y-directions configured as symmetric and anti-symmetric boundary conditions in order to create a periodic array, while the z-direction is configured as a Perfectly Matched Layer (PML). A broadband $(300-1100 \mathrm{~nm})$ plane-wave light source is injected from the top of the simulation domain. A two-dimensional z-normal frequency-domain power monitor is positioned $150 \mathrm{~nm}$ above the light source to extract reflectance and absorption spectra, while a three-dimensional frequency domain power monitor is positioned around the amorphous silicon absorber layer to measure electrical generation rate and spatial carrier generation profiles.

\section{B. Method}

The device structure features an array of nano-scale particles in the form of spheres or flat discs, which are positioned within Indium Tin Oxide (ITO) material at the rear next to the metallic reflector. The reflector material is varied in each investigation, with both $\mathrm{Al}$ and $\mathrm{Ag}$ simulated while the particle remains Ag. Each set of investigations are performed for all topographies, varying particle radius, height, and the distance from the absorber layer and reflector or surface. In this paper, a set of sphere radius results are presented from the rear particle data set. A close-up view of the parameters to be varied within the simulation is shown in figure 2 .
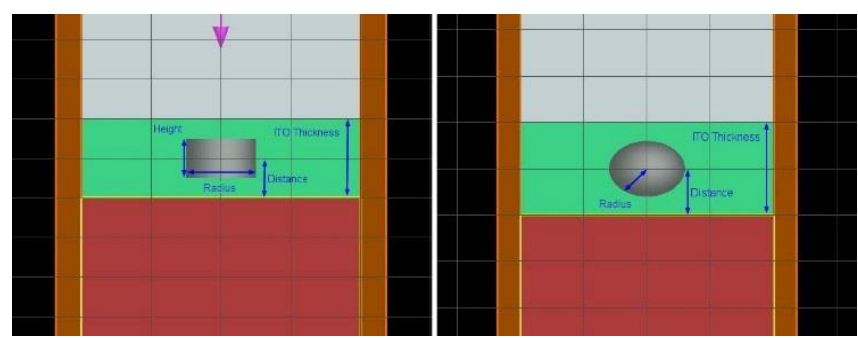

Figure 2 - Schematic diagram illustrating the parameters varied in the simulation

In order to determine the optimum topography for the nanoparticle arrays, optical reflectance from the device structure or carrier generation profile within the active region can be simulated. Two-dimensional optical reflectance simulations require significantly fewer computing resources such as memory and CPU time than three-dimensional generation rate profiles, therefore a wide array of reflectance simulations are performed to provide a rough order of magnitude regarding the desired topography, followed by a reduced number of detailed generation profile simulations to determine the precise optimum.

To determine if an improvement is achieved over an elementary structure without such nano-particle structures, a base-line simulation is performed for each device topography. The results from the baseline simulation, with no embedded nano-particles is overlaid on the figures as dashed lines in the following sections. In order to provide a figure of merit for comparison of each device topology, optical reflectance integrated across the wavelength range is used.

\section{Optical Reflectance Simulation}

The percentage of optical reflectance from the device surface will correspond to a mixture of light reflected at interfaces and unabsorbed light transmitted through the structure reflected back from the metallic reflector. From this measurement an approximation of the amount of light absorbed within the device structure can be calculated, which includes that from within both active and inactive regions.
The positioning of the particles at the rear surface of the device structure causes interactions with light which has passed through the active region from the front surface, and is therefore likely to be light in the longer wavelength red and infra-red regions of the spectrum. The scattering effect can therefore be optimised for this wavelength range to enhance the absorption when light is re-emitted or reflected by the metallic reflector at the rear surface.

\section{1) Sphere Radius Variation in 200nm ITO}

As an example, the simulation results of the metallic sphere radius variation, between $20-100 \mathrm{~nm}$ in steps of $5 \mathrm{~nm}$, are shown in figure 3 for $\mathrm{Ag}$ (a) and $\mathrm{Al}$ (b) back reflector material. The particles are embedded centrally within $200 \mathrm{~nm}$ of ITO adjacent to the back reflector in this investigation, as described in figure 1. Figure 4 depicts the reflectance results for each sphere radius, in addition to the baseline containing no particles depicted in dashed lines for $\mathrm{Ag}$ and $\mathrm{Al}$ materials.
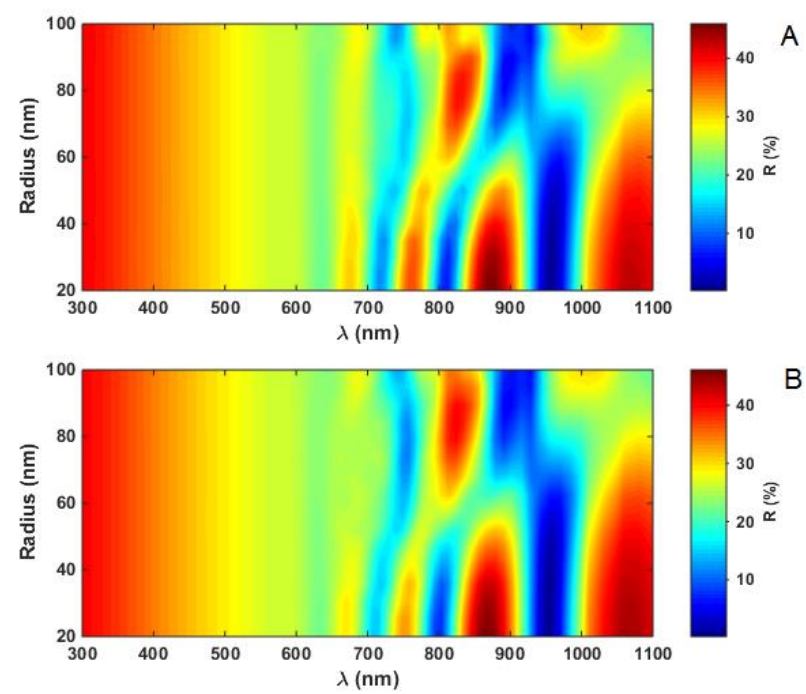

Figure 3 - Reflectance results per wavelength for variation of sphere radius within $200 \mathrm{~nm}$ ITO material for for $\mathrm{Ag}$ (a) and $\mathrm{Al}$ (b) reflector.

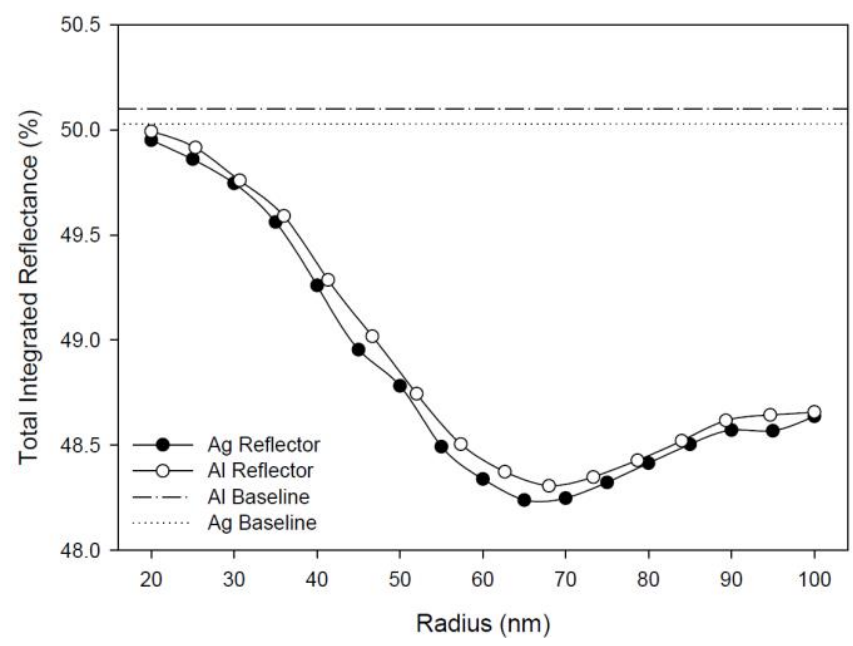

Figure 4 - Plot of total integrated reflectance across all wavelengths vs. sphere radius within $200 \mathrm{~nm}$ ITO material at the rear of the device. 


\section{2) Summary of Results}

The optimum results from each set of parameter sweeps, for both discs and spheres positioned at the rear of the device are detailed in table 1, with the value of minimum reflection observed and corresponding dimension value.

\begin{tabular}{|c|c|c|c|c|c|}
\hline Particle & $\begin{array}{l}\text { Reflector } \\
\text { Material }\end{array}$ & $\begin{array}{l}\text { ITO } \\
\text { Thickness }\end{array}$ & Variable & Value & $\begin{array}{l}\text { Integrated } \\
\text { Reflectance }\end{array}$ \\
\hline Discs & $\mathrm{Al}$ & $100 \mathrm{~nm}$ & Radius & $67 \mathrm{~nm}$ & $52.39 \%$ \\
\hline Discs & $\mathrm{Ag}$ & $200 \mathrm{~nm}$ & Radius & $80 \mathrm{~nm}$ & $48.26 \%$ \\
\hline Discs & $\mathrm{Ag}$ & $200 \mathrm{~nm}$ & Distance & $50 \mathrm{~nm}$ & $48.51 \%$ \\
\hline Discs & $\mathrm{Ag}$ & $200 \mathrm{~nm}$ & Height & $150 \mathrm{~nm}$ & $48.97 \%$ \\
\hline Spheres & $\mathrm{Al}$ & $100 \mathrm{~nm}$ & Radius & $50 \mathrm{~nm}$ & $53.22 \%$ \\
\hline Spheres & $\mathrm{Ag}$ & $200 \mathrm{~nm}$ & Radius & $65 \mathrm{~nm}$ & $48.24 \%$ \\
\hline Spheres & $\mathrm{Ag}$ & $200 \mathrm{~nm}$ & Distance & $110 \mathrm{~nm}$ & $48.63 \%$ \\
\hline Spheres & $\mathrm{Al}$ & $300 \mathrm{~nm}$ & Radius & $20 \mathrm{~nm}$ & $50.48 \%$ \\
\hline Spheres & $\mathrm{Al}$ & $300 \mathrm{~nm}$ & Distance & $200 \mathrm{~nm}$ & $48.97 \%$ \\
\hline
\end{tabular}

Table 1 - Optimum reflectance results from simulation parameter sweeps of $\mathrm{Al}$ and $\mathrm{Ag}$ reflector material

\section{3) Analysis}

As can be observed from the results in table 1, the lowest value of integrated reflectance for particle arrays at the rear of the absorber layer is achieved using spherical Ag particles of radius $65 \mathrm{~nm}$ within $200 \mathrm{~nm}$ ITO and a silver reflector. The value of integrated reflectance appears to drop sharply with increasing radius, increasing again slightly for larger particle radii above $70 \mathrm{~nm}$. All values provide an integrated reflectance lower than the baseline. It is shown that the positioning of the particle mid-way between the absorber layer and back reflector results in a slightly reduced reflectance value, although reflectance remains reduced with a silver reflector if the particle is positioned closer to the absorber layer in this simulation.

\section{Power Absorption Simulation}

The optical reflectance simulations in section 1 provide a good approximation of the amount of light absorbed by the device structure, however it is not possible to differentiate between light absorbed in the active or inactive regions and therefore the contribution to charge carrier generation. In order to determine if the topography which results in the lowest value of optical reflectance corresponds to increased charge carrier generation, the optical power absorption within the silicon thin-film is simulated. Values of lowest reflectance in the previous investigation may not be the optimal conditions for charge carrier generation within the absorber layer, as effects such as parasitic absorption and non-radiative decay of plasmon energy can also cause a reduction in optical reflectance. This does not contribute to increased charge carrier generation and is impossible to determine from a reflectance monitor alone.

Power absorption monitoring is a three-dimensional simulation throughout the absorber layer, and therefore the computational resources required to perform the simulations are significantly higher. Due to this constraint, the condition that varies the optical reflectance percentage by the greatest extent, the particle radius, is varied in this simulation. Spherical particles will be in distributed in either the front or rear ITO regions, with radii varied from 50 to $100 \mathrm{~nm}$ in steps of $10 \mathrm{~nm}$ positioned centrally. In this investigation, simulations are also performed with nano-particle arrays positioned at the front of the absorber region for comparison, within a window layer of ITO identical to that of the rear simulation.

\section{1) Sphere Radius Variation in 200nm ITO at Rear.}

The optical power absorption spectrum of the sphere radius variation simulation in $200 \mathrm{~nm}$ ITO, varied between 50 to $100 \mathrm{~nm}$ in steps of $10 \mathrm{~nm}$ is depicted in figure 5 for particles at the rear ITO material.

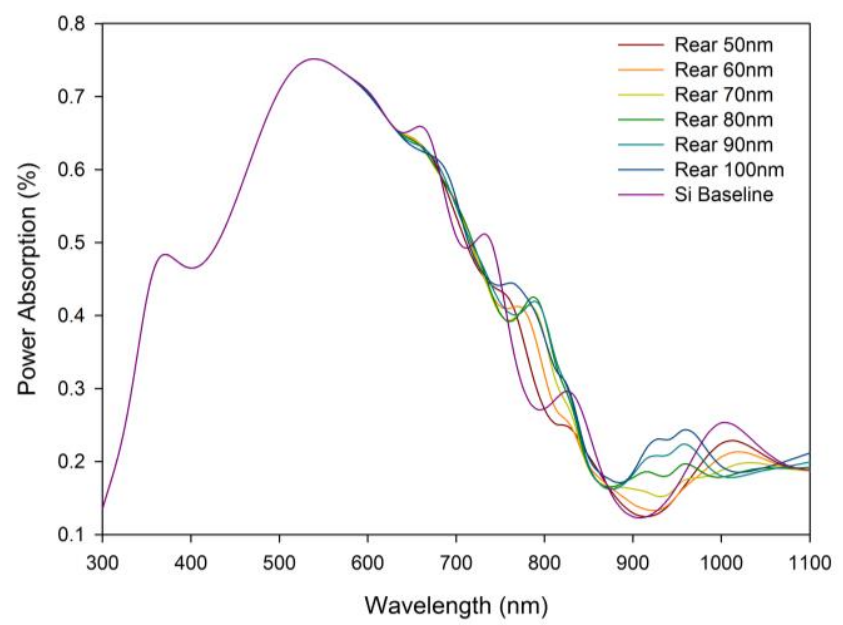

Figure 5 - Plot of power absorption per wavelength from simulation of sphere radius within $200 \mathrm{~nm}$ ITO material at the rear of the device.

The optical power absorption spectrum of the sphere radius variation simulation in $200 \mathrm{~nm}$ ITO, varied between 50 to $100 \mathrm{~nm}$ in steps of $10 \mathrm{~nm}$ is depicted in figure 6 for particles at the front ITO material.

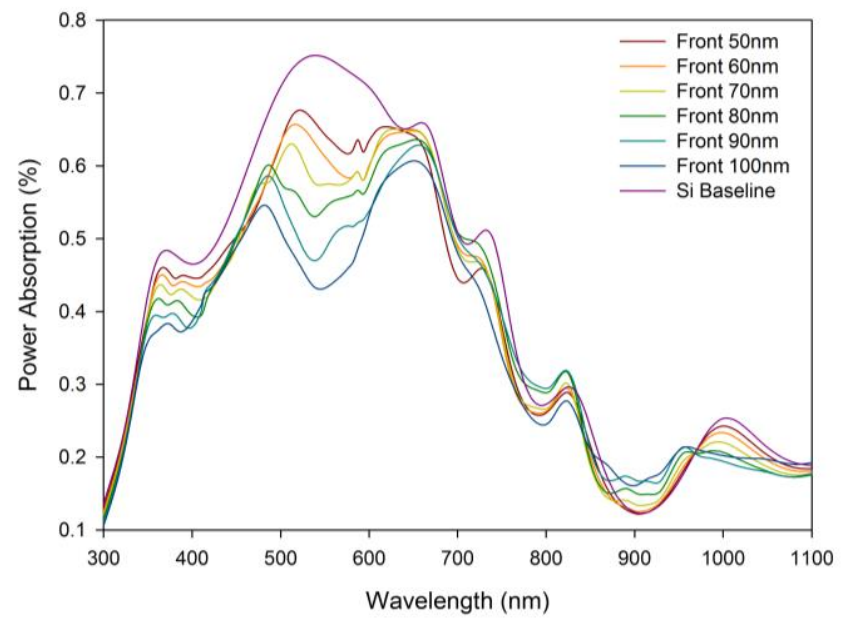

Figure 6 - Plot of power absorption per wavelength from simulation of sphere radius within $200 \mathrm{~nm}$ ITO material at the front of the device.

\section{2) Summary of Results}

Figure 7 depicts the simulated values of optical power absorption within the amorphous silicon region for each value of particle radius. Table 2 details the simulated results for rear particles with the percentage improvement or reduction in power absorption versus baseline with no particles.

\begin{tabular}{llll}
\hline Position & Radius & $\begin{array}{l}\text { Power Absorption } \\
\text { (\%) }\end{array}$ & $\begin{array}{l}\text { Improvement } \\
\text { (\%) }\end{array}$ \\
\hline Rear & $50 \mathrm{~nm}$ & 72.45 & -0.48 \\
\hline Rear & $60 \mathrm{~nm}$ & 72.63 & -0.22 \\
\hline Rear & $70 \mathrm{~nm}$ & 72.8 & $\mathbf{0 . 0 1}$ \\
\hline Rear & $80 \mathrm{~nm}$ & 73.05 & $\mathbf{0 . 3 5}$ \\
\hline Rear & $90 \mathrm{~nm}$ & 73.24 & $\mathbf{0 . 6 2}$ \\
\hline Rear & $100 \mathrm{~nm}$ & 73.59 & $\mathbf{1 . 0 1}$ \\
\hline Si & None & 72.79 & - \\
\hline
\end{tabular}

Table 2 - Power Absorption Simulation Results 


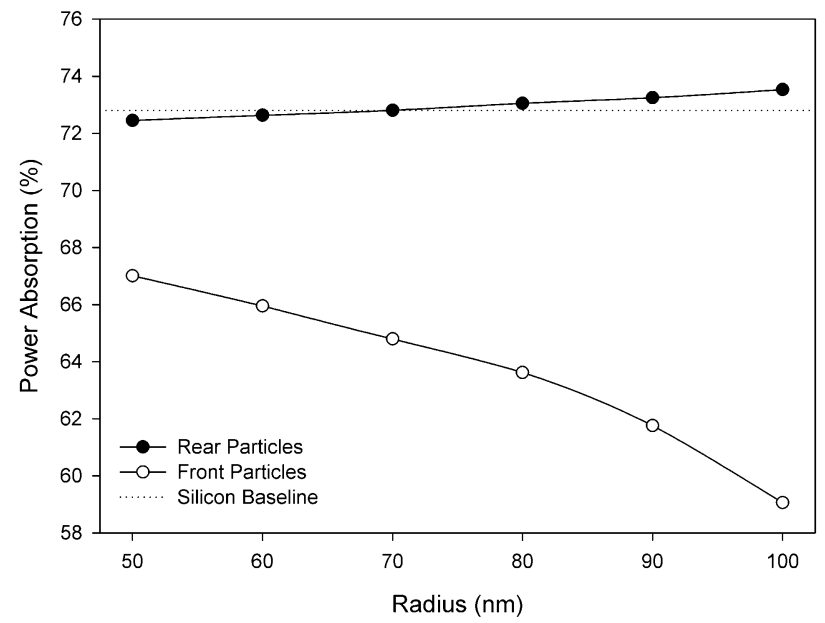

Figure 7 - Plot of power absorption across all wavelengths vs. sphere radius within $200 \mathrm{~nm}$ ITO material for particles at front and rear.

\section{3) Analysis}

The results of power absorption simulations for particles positioned within the rear ITO of the device, shown in figure 7 , are demonstrated to enhance optical power absorption for particle radii in excess of $80 \mathrm{~nm}$. An increase in optical absorption by $1 \%$ or more is achieved for particle radii of $100 \mathrm{~nm}$, while particle radii below $70 \mathrm{~nm}$ cause a slight reduction. Power absorption continues to increase up to the maximum radius of $100 \mathrm{~nm}$, after which point the ITO region will no longer accommodate the particle. Therefore, nanoparticle arrays within the ITO adjacent to the back reflector of the device can enhance optical performance. It can additionally be seen from figure 5 that no optical loss occurs in the short wavelength region from $450-600 \mathrm{~nm}$, whilst power absorption in the longer wavelength region that is weakly absorbed by the silicon shows an enhancement compared to devices with no nano-particle arrays.

Significant losses are evident with the particles mounted at the front as shown in figure 6 . It can be observed that all such particle arrays negatively affect power absorption by a significant degree, with the lowest power absorption loss of just under $8 \%$. Particle radii of increasing size serve to reduce the absorption further so it is reasonable to deduce that front mounted particles are detrimental to device performance with this device configuration.

\section{COMPARISON WITH FABRICATED DEVICES}

\section{A. Process Development of Metal-Island Films}

The particle arrays are formed by the initial deposition of $15 \mathrm{~nm} \mathrm{Ag}$ and $\mathrm{Al}$ material on the surface of a 300nm intrinsic amorphous device layer on glass substrate. Amorphous silicon deposition is performed as per the optimum conditions for intrinsic films detailed in [6-10]. The metal deposition is performed using a Leybold Optics BAK600 electron-beam evaporator system, at a deposition rate of $2.5 \AA / \mathrm{s}(\mathrm{Al})$ and $0.5 \AA / s$ (Ag). This forms a contiguous layer, which is subsequently annealed at temperatures from $200^{\circ} \mathrm{C}$ to $300^{\circ} \mathrm{C}$ for between 30 and 90 minutes using a Jipelec JetFirst 200C rapid thermal annealer to form the metal-island films. Metal island dimensions are shown to be a function of the anneal temperature and duration, therefore a wide array of conditions are evaluated and measured. Figure 8 shows an SEM image, taken using a JEOL JSM-7500F microscope operating at $5 \mathrm{kV}$ with secondary electron imaging detection, showing the resultant nano-particle arrays on the $\mathrm{Si}$ absorber layer after annealling at $300^{\circ} \mathrm{C}$ for 60 minutes.

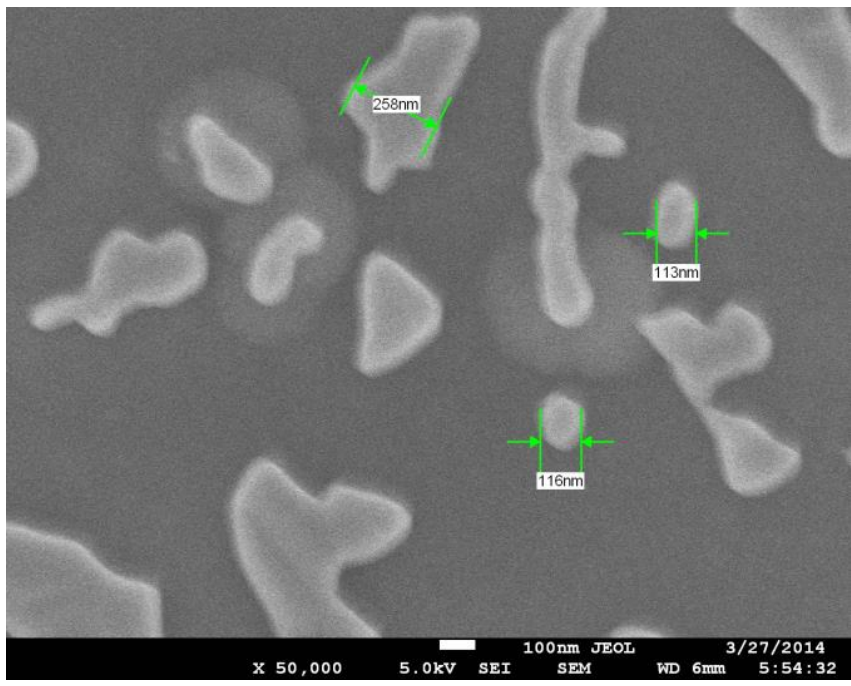

Figure 8 - SEM Image of Ag Particles at 60 Minutes on Si coated glass.

As can be seen from figure 8 , the particles have a wide distribution of dimensions, some of which are circular and some of which are a variety of shapes and sizes. The effects on the incident light are therefore expected to be broad-band compared with the simulated results which feature consistent dimensions. This is due to the various resonant properties of the individual particles and their interactions with the incident light.

\section{B. Amorphous P-I-N Devices}

Following the process development measurements of metal-island films, an amorphous silicon P-I-N device is fabricated using PECVD followed by the deposition and optimum anneal processing of the metal-island film layers. The deposition is performed using an Oxford Instruments PlasmaLab System 100 deposition system, operating at RF 13.56MHz using the following deposition conditions:

\begin{tabular}{lll}
\hline Variable & Unit & Value \\
\hline RF Power & $($ W $)$ & 10 \\
\hline Chamber Pressure & $(\mathrm{mTorr})$ & 350 \\
\hline Substrate Temperature & $\left({ }^{\circ} \mathrm{C}\right)$ & 250 \\
\hline Gas Flow Rate (Silane) & $(\mathrm{sccm})$ & 50 \\
\hline Gas Flow Rate (Hydrogen) & $(\mathrm{sccm})$ & 50 \\
\hline Gas Flow Rate (Diborane) & $(\mathrm{sccm})$ & 10 \\
\hline Gas Flow Rate (Phosphine) & $(\mathrm{sccm})$ & 6 \\
\hline
\end{tabular}

Table 3 - Amorphous Silicon Deposition Conditions taken from [11] 
The substrate material is Pilkington TEC-15 TCO-coated glass, which serves as the front contact of the device. This type of TCO material is selected due to is planar topography in contrast to TEC-8 or Asahi U-type which features a textured surface which serves to obscure the measurable light scattering effect of the nano-particle array. The amorphous silicon is unable to endure temperatures in excess of $300^{\circ} \mathrm{C}$ without a loss of the bonded hydrogen and creation of dangling bonds within the film. Additionally, at temperatures in excess of $400^{\circ} \mathrm{C}$, the amorphous silicon begins to re-crystallise and form a nano-crystalline layer which is not desirable in this device. In this investigation, the anneal is performed at $250^{\circ} \mathrm{C}$, a compromise between scattering enhancement and temperature effects on device performance. Deposition condition and layer thickness optimization of the P-I-N device is performed and published elsewhere [11], resulting in a baseline energy-conversion efficiency of $4.75 \%$. Device topography is detailed in figure 9, showing the P-I-N regions and metal-island film location.

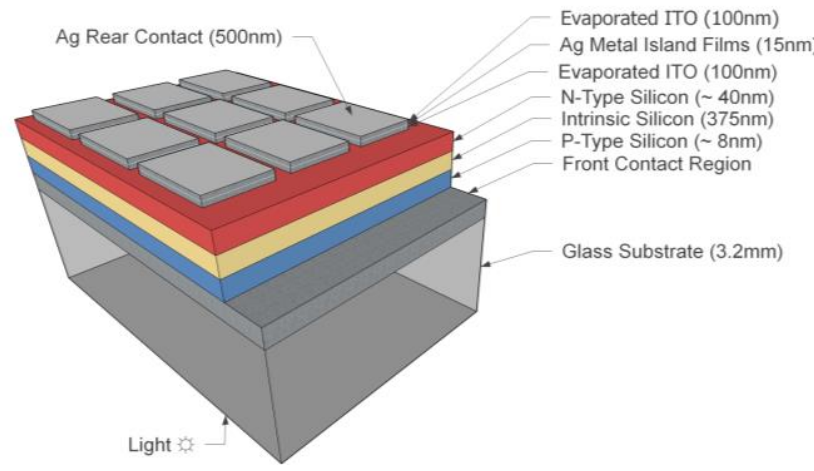

Figure 9 - Topography of P-I-N device with nano-particle array [12]

\section{Measurement and Results}

Measured performance values for devices with and without nano-particle treatments are presented in table 4. Measurement is performed using an Abet technologies SUN 3000 solar simulator configured to project light in the AM1.5 spectrum on to the sample for I-V measurement, and a Bentham Instruments PVE300 configured for spectral range $300-1100 \mathrm{~nm}$ for measurement of quantum efficiency. It can be observed that the P-I-N device featuring the nano-particle array within the back reflector region exhibits a slightly higher value of short-circuit current and maximum-power point current, at the expense of slightly reduced Fill Factor and maximum-power point voltage. This is thought to be due to the slight increase in charge-carrier generation due to enhancement of long-wavelength scattering, while the addition of the silver region in the ITO layer and associated discontinuity within the film are feasible causes of the slight reduction in maximum power point voltage. Overall energy conversion efficiency is shown to be slightly reduced for the device with the nano-particle arrays however this is well within the range of process variation, shown in table 5, and it is therefore not possible to determine if this is due to the addition of the nano-particles. Figures 10 and 11 detail the measured I-V curve and Q-E response of the two P-I-N devices. It can be observed that the I-V responses are virtually identical with no observable difference within the forth quadrant. It can be observed from the external quantum efficiency response that the device featuring nano-particle arrays exhibits a slight increase in quantum efficiency within the range $500-700 \mathrm{~nm}$. It can therefore be deduced that the performance improvement is not localised to a particular wavelength region as is characteristic of uniform nanoparticle arrays, and a broadband enhancement is produced.

\begin{tabular}{lll}
\hline Variable & Baseline Device & Nano-particle Device \\
\hline Efficiency (\%) & 4.75 & 4.66 \\
\hline Fill Factor & 0.58 & 0.56 \\
\hline $\mathrm{V}_{\mathrm{oc}}(\mathrm{V})$ & 0.80 & 0.80 \\
\hline $\mathrm{J}_{\mathrm{sc}}\left(\mathrm{mA} / \mathrm{cm}^{2}\right)$ & 10.11 & 10.28 \\
\hline $\mathrm{V}_{\mathrm{m}}(\mathrm{V})$ & 0.60 & 0.58 \\
\hline $\mathrm{I}_{\mathrm{m}}(\mathrm{mA})$ & 7.91 & 8.04 \\
\hline
\end{tabular}

Table 4 - P-I-N device with nano-particle array parameters

The process variation between alternate devices and regions within the same device, detailed in table 5 , can be attributed to deposition uniformity variations, substrate contamination, plasma edge effects and contact series resistances.

\begin{tabular}{llll}
\hline Region & First Cell & Second Cell & Variation (\%) \\
\hline $\mathbf{1}$ & 5.23 & 5.65 & $7 \%$ \\
\hline $\mathbf{2}$ & 5.20 & 5.52 & $6 \%$ \\
\hline $\mathbf{3}$ & 4.93 & 5.60 & $12 \%$ \\
\hline $\mathbf{4}$ & 4.83 & 5.50 & $12 \%$ \\
\hline $\mathbf{5}$ & 4.93 & 5.52 & $11 \%$ \\
\hline $\mathbf{6}$ & 4.75 & 5.49 & $13 \%$ \\
\hline $\mathbf{7}$ & 4.66 & 4.70 & $1 \%$ \\
\hline $\mathbf{8}$ & 4.62 & 5.19 & $11 \%$ \\
\hline $\mathbf{9}$ & 3.99 & 5.04 & $21 \%$ \\
\hline Average & 4.79 & 5.35 & $10 \%$ \\
\hline \multicolumn{5}{r}{ Table 5 - P-I-N device efficiency variation } \\
between successive depositions
\end{tabular}

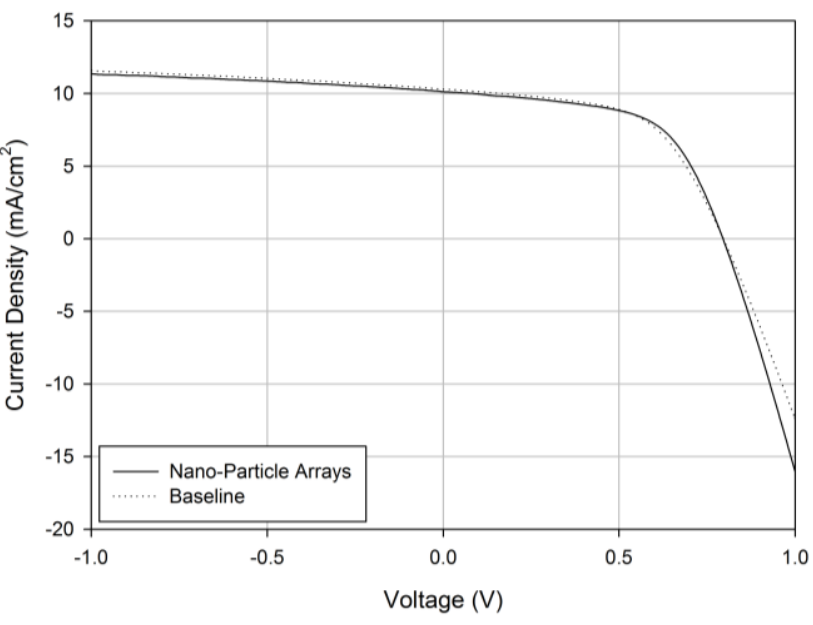

Figure 10 - I-V curve of P-I-N devices with nano-particle arrays and baseline measurement

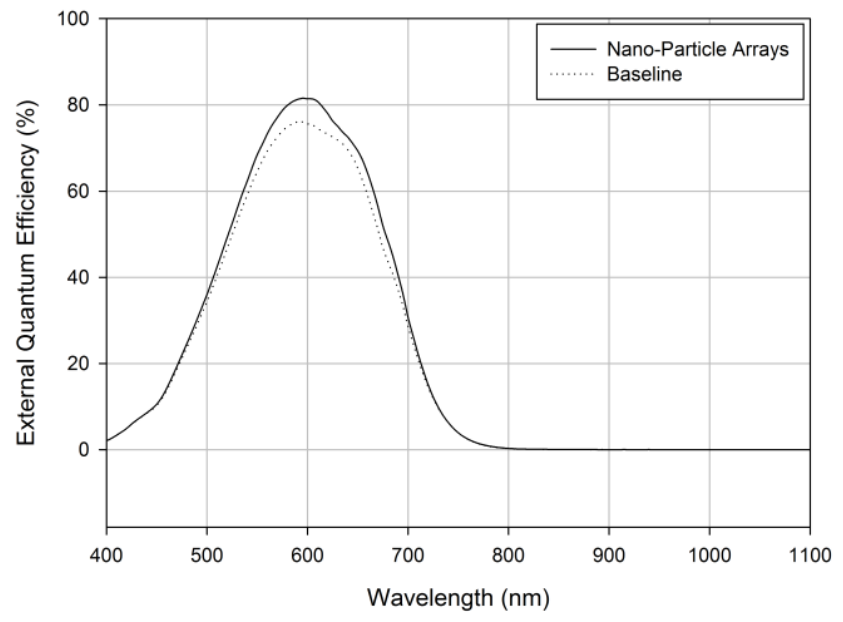

Figure 11 - EQE curve of P-I-N devices with nano-particle arrays and baseline measurement 


\section{CONCLUSION}

It has been demonstrated by means of numerical simulation that the optical absorption and reflection of a silicon thin film with an array of metallic nano-particles can be varied over a wide range. This is achieved by means of adjusting the size, shape, position and constituent material of the surroundings. Positioning of arrays within the rear TCO materials is investigated, together with optimisation of the TCO thickness for these configurations.

The simulations indicate that the optical power absorption in the active layer of an amorphous silicon P-I-N device can be increased by approximately $1 \%$, by the incorporation of $100 \mathrm{~nm}$ radius spherical Ag particle arrays within 200nm rear TCO material adjacent to an Ag back reflector. These serve to scatter transmitted light and therefore increase the effective optical path length through the active layer.

Careful optimisation of the design is required to achieve the enhancement in performance to avoid parasitic absorption and reduction in performance. It is noted that enhancement due to light scattering at the rear of the device is strongly dependent on the absorber layer thickeness, and therefore it is expected that the potential enhancement due to methods simulated in this work are likely to improve with a reduction in absorber layer thickness.

A series of Ag nano-particle arrays fabricated by deposition and annealing of $\mathrm{Ag}$ on a-Si:H thin films has been performed, and their optical properties examined. These nano-oparticle arrays are subsequently integrated into the back reflector region of an amorphous silicon P-I-N solar cell.

Overall efficiency values show a variation within the range $1 \%$, which is within the process variation margin of the fabrication process. It is therefore not possible to distinguish any performance improvement from this value alone, and greater control over experimental conditions are required prove this technique experimentally.

\section{REFERENCES}

[1] R. Brendel. Appendix A: Light Trapping. In Thin-Film Crystalline Silicon Solar Cells, pages $181\{208$. Wiley-VCH Verlag GmbH \& Co. KGaA, 2005.

[2] M. Fonrodona, S. Santos, C. Mata, M. Vetter, and J. Andreu. Performance and productivity improvements in very large area amorphous silicon modules. 25 ${ }^{\text {th }}$ European Photovoltaic Solar Energy Conference (25th PVSEC), 2(September) 7-10, 2010.

[3] H. Maurus, M. Schmid, B. Blersch, P. Lechner, and H. Schade. PV for buildings: Benefits and experiences with amorphous silicon in BIPV applications. Refocus, 5(6):22\{27, 2004.

[4] E. Yablonovitch and G. D. Cody. Intensity enhancement in textured optical sheets for solar cells. Electron Devices, IEEE Transactions on, 29(2):300\{305, 1982.

[5] Antonio Luque, The confinement of light in solar cells, Solar Energy Materials, Volume 23, Issue 2, 1991, Pages 152-163,

[6] S. A. Maier. Plasmonics: Fundamentals and Applications. Springer US, 2007.

[7] V. E. Ferry, J. N. Munday, and H. A. Atwater, "Design considerations for plasmonic photovoltaics," Adv. Mater. 22(43), 4794-4808 (2010).
[8] K. Catchpole and A. Polman, "Design principles for particle plasmon enhanced solar cells," Appl. Phys. Lett. 93(19), 191113 (2008).

[9] H. A. Atwater and A. Polman, "Plasmonics for improved photovoltaic devices," Nat. Mater. 9(3), 205-213 (2010).

[10] M. A. Green and S. Pillai, "Harnessing plasmonics for solar cells," Nat. Photonics 6(3), 130-132 (2012).

[11] L. J. Crudgington, M. A. Rind, D. N. R. Payne, and D. M. Bagnall. The Effects of Varied Deposition Conditions, Including the Use of Argon, on Thin-Film Silicon Solar Cells Prepared Using PECVD. Molecular Crystals and Liquid Crystals, (April 15), 2014.

[12] Crudgington, Lee. High-performance amorphous silicon solar cells with plasmonic light scattering. University of Southampton, Physical Sciences and Engineering, Doctoral Thesis (Dec 2015), 204pp

[13] Lumerical Solutions Inc, FDTD Solutions, 2016.

[14] Edward D. Palik, Handbook of Optical Constants of Solids, Academic Press, Burlington, 2002, ISBN 9780125444156 